Supporting Information to accompany:

\title{
Ligand Conjugation Directs Formation of a 1,3-Dihydropyridinate Regioisomer
}

Tobias J. Sherbow, Leo W. T. Parsons, Nathan A. Phan, James C. Fettinger, and Louise A. Berben*

Department of Chemistry, One Shields Ave, University of California, Davis CA 95616

e-mail: laberben@ucdavis.edu 


\section{Materials and Methods}

Physical Measurements. Cyclic voltammograms (CVs) were recorded in a glove box under $\mathrm{N}_{2}$ (Praxair, 99.998\%). A CH Instruments Electrochemical Analyzer Model 620D was used for electrochemical measurements with a glassy carbon working electrode ( $\mathrm{CH}$ Instruments, nominal surface area of $0.071 \mathrm{~cm}^{2}$ ), a platinum wire auxiliary electrode (BASi), and a $\mathrm{Ag} / \mathrm{AgNO}_{3}(0.001 \mathrm{M})$ non-aqueous reference electrode with a Vycor tip. All potentials are referenced to the SCE couple, and ferrocene was used as an internal standard where the $\mathrm{E}_{1 / 2}$ of ferrocene/ferrocenium is $+0.56 \mathrm{~V}$ vs. SCE in $0.3 \mathrm{M} \mathrm{Bu}_{4} \mathrm{NPF}_{6}$ THF. ${ }^{1}$ Electrolyte solutions were stored over $3 \AA$ molecular sieves for at least 48 hours before use. Elemental analyses were performed by the Microanalytical Laboratory at The University of California, Berkeley. ${ }^{1} \mathrm{H}-\mathrm{NMR}$ and ${ }^{13} \mathrm{C}$-NMR spectra were recorded at ambient temperature using a Varian $600 \mathrm{MHz}$ or $400 \mathrm{MHz}$ spectrometer. Chemical shifts were referenced to residual solvent.

X-ray diffraction studies were carried out on a Bruker SMART APEX Duo or a Bruker Kappa Duo diffractometer equipped with a CCD detector. ${ }^{2}$ Measurements were carried out at $-175^{\circ} \mathrm{C}$ using $\mathrm{Cu} \mathrm{Ka}$ $(1.54178 \AA$ ) radiation. Crystals were mounted on a glass capillary or Kaptan Loop with Paratone-N oil. Initial lattice parameters were obtained from a least-squares analysis of more than 100 centered reflections; these parameters were later refined against all data. Data was integrated and corrected for Lorentz polarization effects using SAINT, ${ }^{3}$ and corrected for absorption effects using SADABS2.3. ${ }^{4}$ Space group assignments were based upon systematic absences, E statistics, and successful refinement of the structures. Structures were solved by direct methods with the aid of successive difference Fourier maps and refined against all data using the SHELXTL 2014/7 software package. ${ }^{5}$ Thermal parameters for all non-hydrogen atoms were refined anisotropically. Hydrogen atoms, where added and assigned to ideal positions and refined using a riding model with an isotropic thermal parameter 1.2 times that of the attached carbon atom (1.5 times for methyl hydrogens).

Preparation of Compounds. All manipulations were carried out under a dinitrogen atmosphere using standard Schlenk line and glovebox techniques. All chemicals were purchased from VWR International, Acros, Alpha Aesar, or Cambridge Isotopes and used as received unless otherwise noted. Bulk solvents were deoxygenated and dried by sparging with argon gas followed by passage through an activated alumina column. Deuterated solvents and other liquid reagents were degassed with dinitrogen and stored over activated $3 \AA$ sieves prior to use. $\left(\mathrm{I}_{2} \mathrm{P}^{2-}\right) \mathrm{AICl}$ (1) was synthesized in accordance with a reported procedure. ${ }^{6}$

$\left({ }^{\left({ }^{2}\right.} \mathbf{I}_{2} \mathrm{P}^{-}\right) \mathrm{Al}($ TEMPO)Cl (2). Compound 1 (157 mg, $0.23 \mathrm{mmol})$ was dissolved in benzene $(5 \mathrm{~mL})$ in a scintillation vial in a nitrogen filled glovebox. 0.95 equivalents of TEMPO (34 $\mathrm{mg}, 0.22 \mathrm{mmol}$ ) dissolved in benzene $(2 \mathrm{~mL})$ was then added to the solution of 1 . The reaction solution turned from an orange brown solution to red after stirring for one minute, the benzene solution was filtered through Celite and the solvent was removed in vacuo. Compound 2 was collected as a red powder ( $189 \mathrm{mg}, 95 \%$ yield). Single crystals of 2 were grown as red plates from a concentrated hexane solution held at $-25^{\circ} \mathrm{C}$ overnight. Anal. Calcd. For $\mathrm{C}_{52} \mathrm{H}_{65} \mathrm{AlN}_{4} \mathrm{O}$ : C, 75.75; H, 7.95; N, 6.79. Found: C, 75.09; H, 7.88; N, 6.69. Evan's Method: $\mu_{\text {eff }}=1.63 \mu_{\mathrm{B}}$ at $298 \mathrm{~K}$.

$\left({ }^{\left(\mathrm{h}_{2}\right.} \mathrm{P}^{2-}\right) \mathrm{Al}($ TEMPO) (3). Compound $2(660 \mathrm{mg}, 1.04 \mathrm{mmol})$ was dissolved in benzene $(5 \mathrm{~mL})$ in a scintillation vial in a nitrogen filled glovebox. One equivalent of $\mathrm{Na}(23 \mathrm{mg}, 1.00 \mathrm{mmol})$ was added to the solution of 2 and stirred for $48 \mathrm{hrs}$. The reaction solution turned from red to dark brown-yellow over 48 hrs. The benzene solution was then filtered through Celite to remove $\mathrm{NaCl}$ and the solvent was removed in vacuo. Compound 3 was collected as a brown powder (496 mg, 68\% yield). Single crystals of complex 3 were grown as large brown plates from a concentrated hexane solution held at $-25{ }^{\circ} \mathrm{C}$ overnight. ${ }^{1} \mathrm{H}$ NMR (400 MHz, $\left.\mathrm{C}_{6} \mathrm{D}_{6}\right): \delta$ 7.10-6.90 (m, 18H, Ar, m-py), 5.79 (t, 1H, J= 7.2 Hz p-py), 2.85 (br m, 4H, $\left.\mathrm{CH}\left(\mathrm{CH}_{3}\right)_{2}\right), 1.51\left(\mathrm{~d}, 12 \mathrm{H}, \mathrm{J}=5.8 \mathrm{~Hz}, \mathrm{CH}\left(\mathrm{CH}_{3}\right)_{2}\right), 1.18\left(\mathrm{~d}, 12 \mathrm{H}, \mathrm{J}=6.2 \mathrm{~Hz}, \mathrm{CH}\left(\mathrm{CH}_{3}\right)_{2}\right), 1.01(\mathrm{~m}, 14 \mathrm{H}$, TEMPO), 0.57 (br m, 4H, TEMPO). ${ }^{13} \mathrm{C}-\mathrm{NMR}\left(151 \mathrm{MHz}, \mathrm{C}_{6} \mathrm{D}_{6}\right.$ ): $\delta$ 143.90, 130.06, 126.48, 124.73, 123.82, 
$120.53,59.39,40.23,33.06,29.26,28.09,25.88,25.61,24.73,24.47,20.52,18.08$. Anal. Calcd. For $\mathrm{C}_{52} \mathrm{H}_{65} \mathrm{AIN}_{4} \mathrm{O}: \mathrm{C}, 79.15 ; \mathrm{H}, 8.30 ; \mathrm{N}, 7.10$. Found: C, 78.82; $\mathrm{H}, 8.44 ; \mathrm{N}, 7.46$.

[K(18-C-6)(THF) $\left.)_{2}\right]\left[\left(\mathrm{HI}_{2} \mathrm{P}^{3-}\right) \mathrm{Al}(\mathrm{TEMPO})\right]$ (4). Synthesis using $\mathrm{K}$ as a reductant: Compound 2 $(77.5 \mathrm{mg}, 0.089 \mathrm{mmol})$ was dissolved in THF $(5 \mathrm{~mL})$ in a scintillation vial in a nitrogen filled glovebox. Three equivalents of $\mathrm{K}(10.8 \mathrm{mg}, 0.28 \mathrm{mmol})$ and one equivalent of $18-\mathrm{C}-6(24.0 \mathrm{mg}, 0.091 \mathrm{mmol})$ was added to the solution of 2 while stirring. The reaction solution turned from red to dark yellow-brown over $12 \mathrm{hrs}$. The THF was removed in vacuo and the residual powder was triturated with benzene. The suspension was filtered through Celite leaving a brown powder on top of the Celite which was extracted with $3 \mathrm{~mL}$ THF and $2 \mathrm{~mL}$ of hexane was layered on top and placed in a freezer to be held at $-25^{\circ} \mathrm{C}$ overnight. Large brown crystals of 4 were obtained (97 mg, 82\% yield).

Synthesis using KH as hydride source: Compound 2 (70.8 $\mathrm{mg}, 0.093 \mathrm{mmol})$ was dissolved in THF (5 mL) in a scintillation vial in a nitrogen filled glovebox. $\mathrm{KH}(3.7 \mathrm{mg}, 0.093 \mathrm{mmol})$ and one equivalent of $18-\mathrm{C}-6(23.0 \mathrm{mg}, 0.087 \mathrm{mmol})$ was added to the solution which was stirred overnight. The solution turned from a brown-yellow color to a deeper brown-yellow color. The THF was removed in vacuo and the residual powder was triturated with benzene. The suspension was filtered through Celite leaving a brown powder on top of the Celite which was extracted with $3 \mathrm{~mL}$ THF to give $4(20 \mathrm{mg}, 18 \%$ yield). ${ }^{1} \mathrm{H}-\mathrm{NMR}\left(600 \mathrm{MHz}, d_{8}-\mathrm{THF}\right): \delta 7.02-6.45(\mathrm{~m}, 16 \mathrm{H}, \mathrm{Ar}), 6.36(\mathrm{~d}, 1 \mathrm{H}, J=9.7 \mathrm{~Hz} o-p y), 5.06(\mathrm{~m}, 1 \mathrm{H}$, p-py), 4.65 (hept, $\left.1 \mathrm{H}, J=8.0 \mathrm{~Hz}, \mathrm{CH}\left(\mathrm{CH}_{3}\right)_{2}\right), 3.75$ (hept, $\left.1 \mathrm{H}, J=6.8 \mathrm{~Hz}, \mathrm{CH}\left(\mathrm{CH}_{3}\right)_{2}\right), 3.57$ (m, 33H, 18-C-6, THF, $\left.\mathrm{CH}\left(\mathrm{CH}_{3}\right)_{2}\right), 3.38$ (m 1H, o-py), 3.34 (hept, $\left.1 \mathrm{H}, \mathrm{J}=6.7 \mathrm{~Hz}, \mathrm{CH}\left(\mathrm{CH}_{3}\right)_{2}\right), 2.77$ (dd, $1 \mathrm{H}, J=17.90,6.20$ $\mathrm{Hz}$, o-py), 1.71 (m, 8H, THF), 1.43-0.35 (m, 42H, TEMPO, $\left.\mathrm{CH}\left(\mathrm{CH}_{3}\right)_{2}\right) \cdot{ }^{13} \mathrm{C}-\mathrm{NMR}\left(600 \mathrm{MHz}, \mathrm{d}_{8}-\mathrm{THF}\right):{ }^{13} \mathrm{C}$ NMR $\left(151 \mathrm{MHz}\right.$, THF- $\left.d_{8}\right) \delta 151.22,150.38,148.71,148.68,145.58,143.95,141.25,140.97,139.24$, $131.44,131.19,129.74,129.17,128.51,127.12$, 126.75, 126.70, 126.48, 124.89, 123.54, 123.26, 123.02, $122.59,122.50,120.82,71.26,68.26,58.86,40.61,35.51,32.59,30.67,28.73,28.23,27.90,27.74$, 27.21, 26.43, 23.58, 19.06, 18.24, 14.49. Anal. Calcd. For $\mathrm{C}_{72} \mathrm{H}_{106} \mathrm{AlKN}_{4} \mathrm{O}_{9}: \mathrm{C}, 69.87 ; \mathrm{H}, 8.63 ; \mathrm{N}, 4.53$. Found: C, 69.17; H, 8.14; N, 4.69 . 
Table S1. Crystallographic data for $\left(\mathrm{I}_{2} \mathrm{P}^{-}\right) \mathrm{Al}(\mathrm{TEMPO}) \mathrm{Cl}(2)$, $\left(\mathrm{l}_{2} \mathrm{P}^{2-}\right) \mathrm{Al}(\mathrm{TEMPO})(\mathbf{3})$, and $\left[\left({ }^{\left({ }^{2}\right.} I_{2} \mathrm{P}^{3-}\right) \mathrm{Al}(\mathrm{TEMPO}]\left[\mathrm{K}(18-\mathrm{C}-6)(\mathrm{THF})_{2}\right](4)\right.$.

\begin{tabular}{|c|c|c|c|}
\hline & 2 & 3 & 4 \\
\hline Formula & {$\left[\mathrm{C}_{52} \mathrm{H}_{65} \mathrm{AICIN}{ }_{4} \mathrm{O}\right]_{2}[\mathrm{THF}]_{2.5}$} & $\mathrm{C}_{52} \mathrm{H}_{65} \mathrm{AIN}{ }_{4} \mathrm{O}$ & {$\left[\mathrm{C}_{52} \mathrm{H}_{66} \mathrm{AlN}_{4} \mathrm{O}\right]\left[\mathrm{C}_{20} \mathrm{H}_{40} \mathrm{KO}_{8}\right]$} \\
\hline Crystal size & $0.30 \times 0.19 \times 0.11$ & $0.20 \times 0.15 \times 0.09$ & $0.94 \times 0.70 \times 0.46$ \\
\hline Form wt, gmol ${ }^{-1}$ & 1829.27 & 789.06 & 1237.68 \\
\hline Space group & $P \overline{1}$ & $P \overline{1}$ & $P \overline{1}$ \\
\hline$a, \AA$ & $9.3382(4)$ & $11.6980(2)$ & $13.5224(3)$ \\
\hline$b, \AA$ & $23.2879(11)$ & $12.7736(2)$ & $24.0175(6)$ \\
\hline$c, \AA$ & $23.8172(12)$ & $16.7478(3)$ & $25.5555(7)$ \\
\hline$\alpha$, deg & $91.398(3)$ & $106.4777(8)$ & $80.8871(13)$ \\
\hline$\beta$, deg & $91.185(3)$ & $91.5031(8)$ & $81.0756(12)$ \\
\hline$\gamma$, deg & $100.755(3)$ & $108.3186(8)$ & $78.2721(13)$ \\
\hline$V, \AA^{3}$ & $5085.3(4)$ & $2259.78(7)$ & $7958.8(3)$ \\
\hline$Z$ & 2 & 2 & 4 \\
\hline$T, \mathrm{~K}$ & $90(2)$ & $90(2)$ & $90(2)$ \\
\hline$\rho$, calcd, $\mathrm{g} \mathrm{cm}^{-3}$ & 1.195 & 1.160 & 1.033 \\
\hline Refl. coll/ $2 \theta_{\max }$ & $35688 / 145.28$ & $13815 / 44.596$ & 53578 / 144.85 \\
\hline $\begin{array}{l}\text { Uniq refl./ } I>2 \sigma \\
(I)\end{array}$ & 19024 / 14629 & 8313 / 6942 & $29310 / 26656$ \\
\hline Param./restr. & 1196 / 33 & $535 / 0$ & 1644 / 16 \\
\hline$\lambda, \AA \AA \AA / K \alpha, \mathrm{cm}^{-1} \mathrm{~b}$ & 1.54178 & 1.54178 & 1.54178 \\
\hline $\mathrm{R}_{1} / \mathrm{GOF}$ & $0.0636 / 1.071$ & 0.0382 / 1.017 & $0.0578 / 1.028$ \\
\hline $\mathrm{wR}_{2}(I>2 \sigma(I))^{\mathrm{c}}$ & 0.1780 & 0.0932 & 0.1501 \\
\hline $\begin{array}{l}\text { Residual } \\
\text { density, e } \AA^{-3}\end{array}$ & $1.432 /-0.467$ & $0.279 /-0.309$ & $0.614 /-0.558$ \\
\hline
\end{tabular}

${ }^{a}$ Obtained with graphite-monochromated Mo $\mathrm{K} \alpha(\lambda=0.71073 \AA)$ radiation. ${ }^{b}$ Obtained with graphite-monochromated $\mathrm{Cu} \mathrm{Ka}(\lambda=1.54178 \AA)$ radiation. ${ }^{c} R 1=\Sigma|| F_{\mathrm{o}}\left|-F_{\mathrm{c}}\right| / \Sigma\left|F_{\mathrm{o}}\right|, \mathrm{w} R 2$ $=\left\{\Sigma\left[\mathrm{w}\left(F_{\mathrm{o}}^{2}-F_{\mathrm{c}}^{2}\right)^{2}\right] / \Sigma\left[\mathrm{w}\left(F_{\mathrm{o}}^{2}\right)^{2}\right]\right\}^{1 / 2}$. 
Table S2. Selected average interatomic distances $(\AA)$ and selected average angles (deg) for $\left(\mathrm{I}_{2} \mathrm{P}^{-}\right) \mathrm{Al}(\mathrm{TEMPO}) \mathrm{Cl}(\mathbf{2})(\mathrm{X}=\mathrm{Cl}, \mathrm{Y}=\mathrm{O}),\left(\mathrm{I}_{2} \mathrm{P}^{2-}\right) \mathrm{Al}(\mathrm{TEMPO})(3)(\mathrm{Y}=\mathrm{O})$, and $\left[\left({ }^{\mathrm{Ph}} \mathrm{I}_{2} \mathrm{P}^{3-}\right.\right.$ )Al(TEMPO][K(18-C-6)(THF) $)_{2}$ (4). There are two entries each for 2 and $\mathbf{4}$ because each structure contains two distinct molecules in the asymmetric unit.

\begin{tabular}{|c|c|c|c|c|c|}
\hline & 2 & $\mathbf{2}^{\prime}$ & 3 & 4 & $4^{\prime}$ \\
\hline $\mathrm{Al}-\mathrm{N}_{\mathrm{im}}$ & $2.162(2)$ & $2.119(2)$ & $1.9416(12)$ & $1.875(2)$ & $1.894(2)$ \\
\hline $\mathrm{Al}-\mathrm{N}_{\mathrm{am}}$ & $2.057(2)$ & $2.106(2)$ & $1.8891(12)$ & $1.887(2)$ & $1.888(2)$ \\
\hline $\mathrm{Al}-\mathrm{N}_{\mathrm{py}}$ & $1.905(2)$ & $1.908(2)$ & $1.8312(12)$ & $1.851(2)$ & $1.843(1)$ \\
\hline$M-X$ & $2.1625(9)$ & $2.1651(9)$ & $\mathrm{N} / \mathrm{A}$ & $\mathrm{N} / \mathrm{A}$ & $\mathrm{N} / \mathrm{A}$ \\
\hline$M-Y$ & $1.771(2)$ & $1.765(2)$ & $1.7181(11)$ & $1.742(1)$ & $1.737(1)$ \\
\hline $\mathrm{N}_{\mathrm{im}}-\mathrm{C}_{\mathrm{im}}$ & $1.314(3)$ & $1.336(3)$ & $1.3465(18)$ & $1.416(3)$ & $1.409(2)$ \\
\hline $\mathrm{N}_{\mathrm{am}}-\mathrm{C}_{\mathrm{am}}$ & $1.338(3)$ & $1.327(3)$ & $1.4143(18)$ & $1.454(2)$ & $1.451(2)$ \\
\hline $\mathrm{N}_{p y}-\mathrm{C}_{\mathrm{py}(0)}$ & $1.363(3)$ & $1.365(3)$ & $1.3991(18)$ & $1.402(3)$ & $1.398(3)$ \\
\hline $\mathrm{N}_{p y}-\mathrm{C}_{p y(0)}$ & $1.369(3)$ & $1.358(3)$ & $1.3890(17)$ & $1.404(3)$ & $1.408(3)$ \\
\hline $\mathrm{C}_{\mathrm{py}(0)}-\mathrm{C}_{\mathrm{im}}$ & $1.453(4)$ & $1.453(4)$ & $1.3994(19)$ & $1.354(3)$ & $1.363(3)$ \\
\hline $\mathrm{C}_{\mathrm{am}}-\mathrm{C}_{\mathrm{py}(\mathrm{o})}$ & $1.446(4)$ & 1.447(4) & $1.4427(19)$ & $1.377(3)$ & $1.378(3)$ \\
\hline$C_{p y(m)}-C_{p y(o)}$ & $1.381(4)$ & $1.389(3)$ & $1.406(2)$ & $1.506(3)$ & $1.507(3)$ \\
\hline$C_{p y(o)}-C_{p y(m)}$ & $1.402(4)$ & $1.388(4)$ & $1.377(2)$ & $1.337(3)$ & $1.441(3)$ \\
\hline$C_{p y(p)}-C_{p y(m)}$ & $1.398(4)$ & $1.385(4)$ & $1.384(2)$ & $1.449(3)$ & $1.338(3)$ \\
\hline$C_{p y(p)}-C_{p y\left(m^{\prime}\right)}$ & $1.378(4)$ & $1.389(4)$ & $1.413(2)$ & $1.512(4)$ & $1.515(4)$ \\
\hline $\mathrm{N}_{\mathrm{im}}-\mathrm{M}-\mathrm{N}_{\mathrm{py}}$ & $76.31(9)$ & $76.92(8)$ & $83.64(5)$ & $86.94(8)$ & $87.62(7)$ \\
\hline $\mathrm{N}_{\mathrm{am}}-\mathrm{M}-\mathrm{N}_{\mathrm{py}}$ & $78.11(9)$ & $77.07(8)$ & $86.58(5)$ & $88.57(8)$ & $88.12(7)$ \\
\hline $\mathrm{N}_{\mathrm{am}}-\mathrm{M}-\mathrm{N}_{\mathrm{im}}$ & $145.48(9)$ & $144.25(9)$ & $134.16(5)$ & $128.73(8)$ & $129.91(7)$ \\
\hline $\mathrm{N}_{\mathrm{im}}-\mathrm{M}-\mathrm{X}$ & $91.77(6)$ & $93.59(6)$ & $\mathrm{N} / \mathrm{A}$ & $\mathrm{N} / \mathrm{A}$ & $\mathrm{N} / \mathrm{A}$ \\
\hline $\mathrm{N}_{\mathrm{am}}-\mathrm{M}-\mathrm{X}$ & $95.80(6)$ & $94.39(6)$ & $\mathrm{N} / \mathrm{A}$ & $\mathrm{N} / \mathrm{A}$ & $\mathrm{N} / \mathrm{A}$ \\
\hline $\mathrm{N}_{\mathrm{py}}-\mathrm{M}-\mathrm{X}$ & $144.43(8)$ & $145.94(7)$ & $N / A$ & $\mathrm{~N} / \mathrm{A}$ & $N / A$ \\
\hline $\mathrm{N}_{\mathrm{im}}-\mathrm{M}-\mathrm{Y}$ & $91.06(9)$ & $93.60(9)$ & $98.53(5)$ & $104.04(7)$ & $104.77(7)$ \\
\hline$N_{a m}-M-Y$ & $116.49(9)$ & $115.10(9)$ & $121.98(5)$ & $119.93(7)$ & $119.15(7)$ \\
\hline$N_{p y}-M-Y$ & $101.24(10)$ & $100.82(9)$ & $128.36(5)$ & $124.65(8)$ & $122.59(7)$ \\
\hline
\end{tabular}


Scheme S1. Line drawing showing possible isomers for dihydropyridinates (DHP-s) that are accessible on the $\mathrm{I}_{2} \mathrm{P}$ ligand scaffold in its various charge states. The DHP' isomer that is likely to be most stable is in column A, and less stable in column $B$. The relative stability of the DHP' isomers may also be metal-dependent and that is not explored in this work.
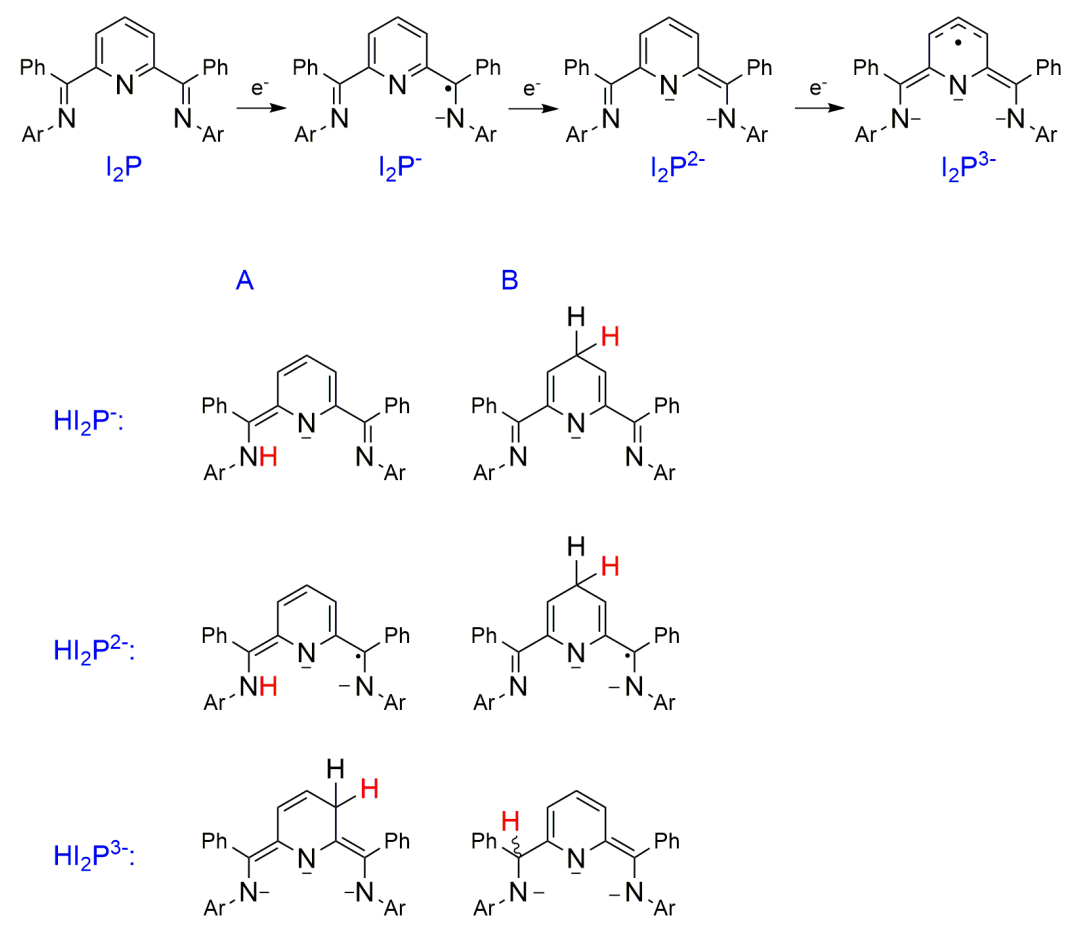


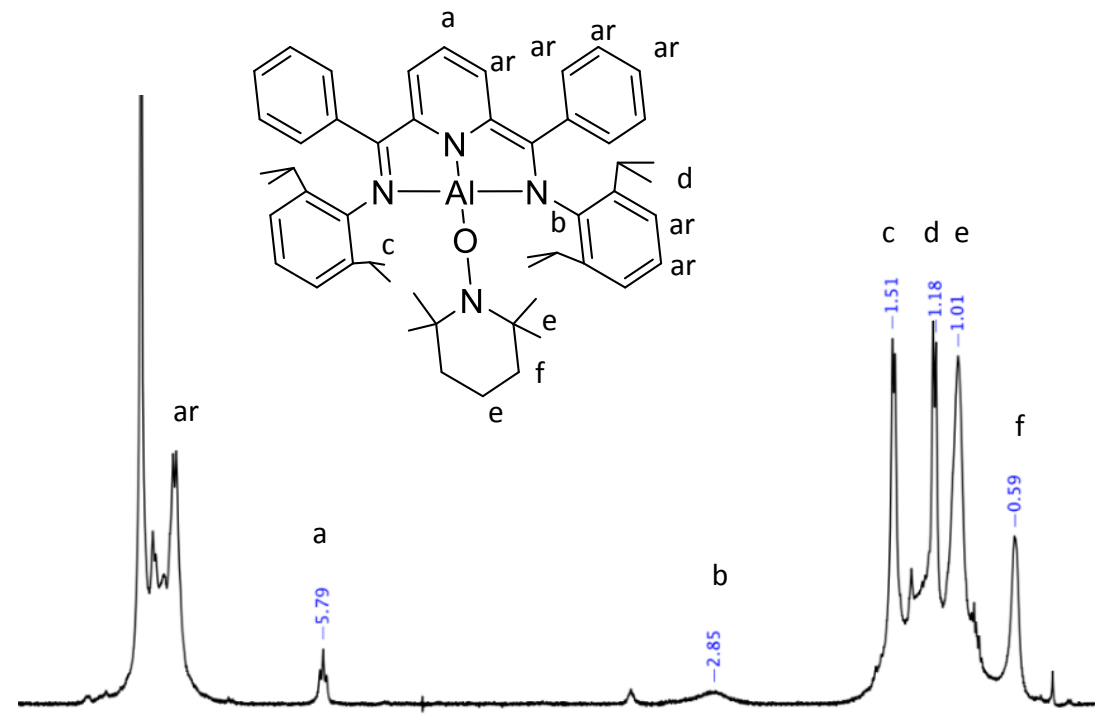

$\begin{array}{llllllllllllllllll}3.0 & 7.5 & 7.0 & 6.5 & 6.0 & 5.5 & 5.0 & 4.5 & 4.0 & 3.5 & 3.0 & 2.5 & 2.0 & 1.5 & 1.0 & 0.5 & 0 .\end{array}$ $\partial(\mathrm{ppm})$

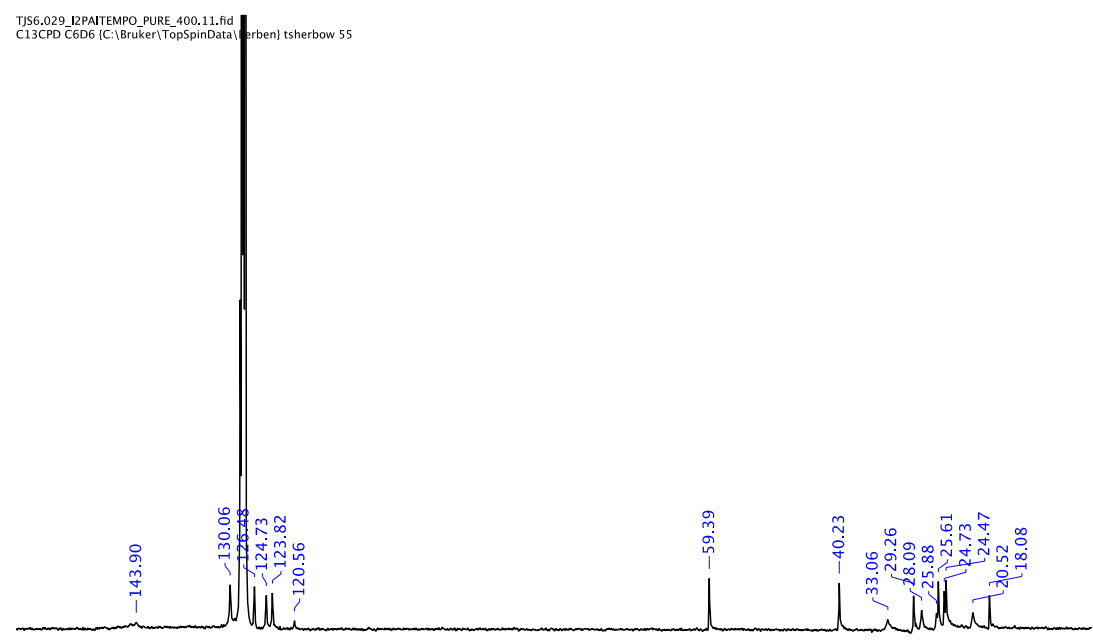

$\begin{array}{llllllllllllllll}60 & 150 & 140 & 130 & 120 & 110 & 100 & 90 & \begin{array}{c}80 \\ \partial(\mathrm{ppm})\end{array} & 70 & 60 & 50 & 40 & 30 & 20 & 10\end{array}$

Figure S1. ${ }^{1} \mathrm{H}$ NMR (top) and ${ }^{13} \mathrm{C}$ spectra (bottom) of $\left(\mathrm{I}_{2} \mathrm{P}^{2-}\right) \mathrm{Al}(\mathrm{TEMPO})(3)$. 

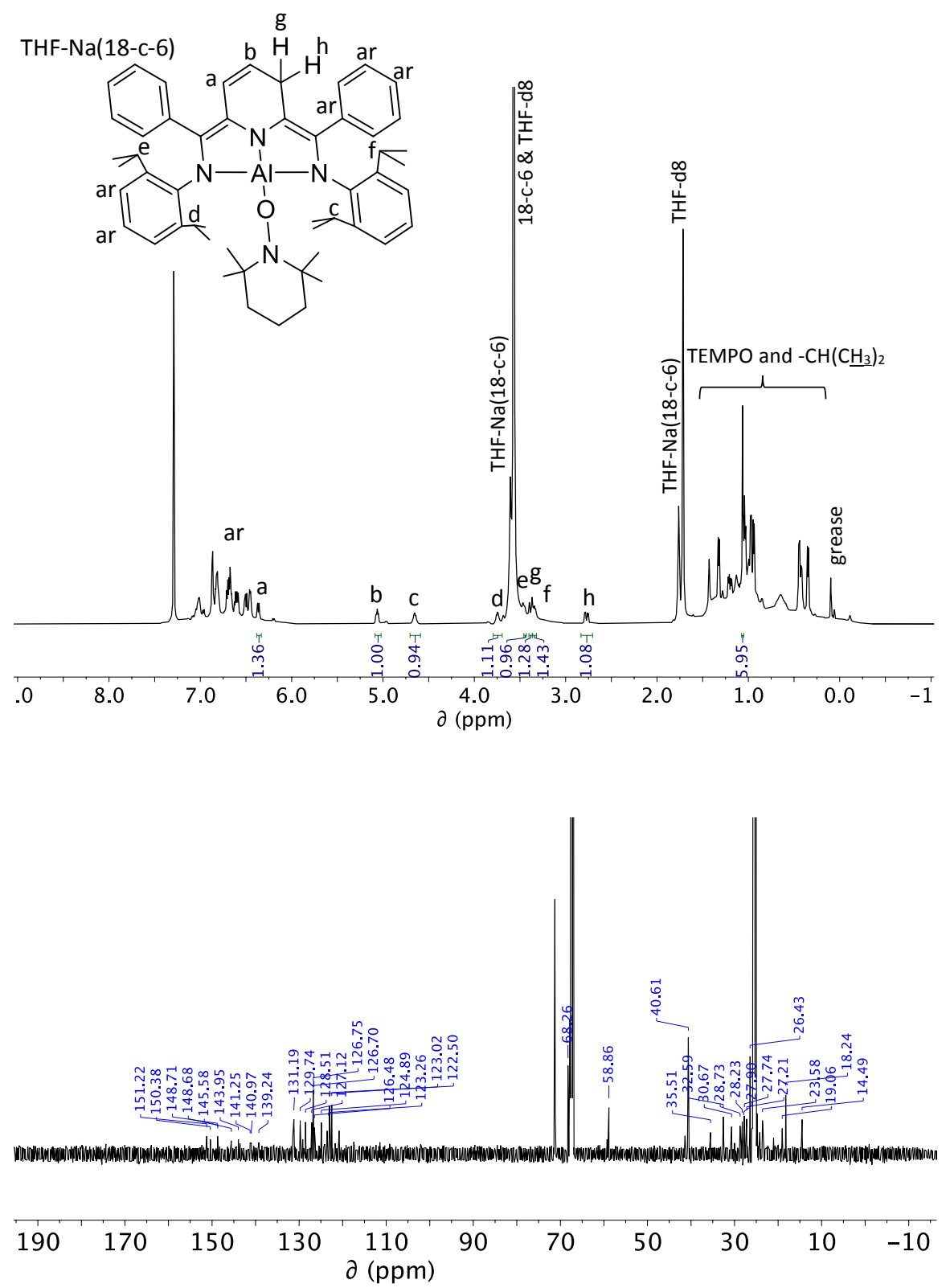

Figure S2. ${ }^{1} \mathrm{H}$ NMR (top) and ${ }^{13} \mathrm{C}$ spectra (bottom) of $\left[\left(\mathrm{Ph}_{2} \mathrm{P}^{3-}\right) \mathrm{Al}(\mathrm{TEMPO}][\mathrm{K}(18-\mathrm{C}-\right.$ 6)(THF) 2 (4).

\section{References}

${ }^{1}$ N. G. Connelly, W. E. Geiger, Chemical Redox Agents for Organometallic Chemistry. Chem. Rev. 1996, 96, 877-910.

${ }^{2}$ SMART Software Users Guide, Version 5.1; Bruker Analytical X-Ray Systems, Inc.: Madison, WI 1999.

${ }^{3}$ SAINT Software Users Guide, Version 7.0; Bruker Analytical X-Ray Systems, Inc; Madison, WI 1999.

${ }^{4}$ Sheldrick, G. M. SADABS, Version 2.03; Bruker Analytical X-Ray Systems, Inc.: Madison, WI, 2000.

${ }^{5}$ Sheldrick, G. M. SHELXTL, Version 6.12; Bruker Analytical X-Ray Systems, Inc.: Madison, WI, 1999.

${ }^{6}$ Thompson, E. J.; Myers, T. W.; Berben, L. A. Synthesis of Square-Planar Aluminum(III) Complexes. Angew. Chem. Int. Ed. 2014, 53, 14132-14134. 